\title{
Observed versus modelled stellar CME rates
}

\author{
Martin Leitzinger ${ }^{1}$, Petra Odert ${ }^{1}$, Krisztian Vida ${ }^{2}$, \\ Levente Kriskovics ${ }^{2}$, Eike W. Guenther ${ }^{3}$, Heidi Korhonen ${ }^{4}$, \\ Robert Greimel $^{1}$, Arnold Hanslmeier ${ }^{1}$ and Helmut Lammer ${ }^{5}$ \\ ${ }^{1}$ University of Graz, Institute of Physics/IGAM, NAWI Graz \\ Universitätsplatz 5, 8010 Graz, Austria \\ email: martin.leitzinger@uni-graz.at \\ ${ }^{2}$ Konkoly Observatory, MTA CSFK, Konkoly Thege Miklós út 15-17, 1121 Budapest, Hungary \\ ${ }^{3}$ Thüringer Landessternwarte Tautenburg, Karl-Schwarzschild-Observatorium, \\ Sternwarte 5, 07778 Tautenburg, Germany \\ ${ }^{4}$ Dark Cosmology Centre, Niels Bohr Institute, University of Copenhagen, \\ Juliane Maries Vej 30, 2100 Copenhagen \\ ${ }^{5}$ Space Research Institute, Austrian Academy of Science, Schmiedlstraße 6, 8042 Graz, Austria
}

\begin{abstract}
Stellar coronal mass ejections (CMEs) may play an important role in stellar and planetary evolution, therefore the knowledge on parameter distributions of this energetic activity phenomenon is highly relevant. During the last years several attempts have been made to detect stellar CMEs of late-type main-sequence and pre main-sequence stars from dedicated optical spectroscopic observations. Up to now only a handful of distinct stellar CME detections are known which contradicts the results from stellar CME modelling, which predict higher CME rates. We report on dedicated ongoing and future observational attempts to detect stellar CMEs and discuss the observational results with respect to the results from stellar CME modelling.
\end{abstract}

Keywords. stars: activity, stars: late-type, stars: chromospheres

To gain knowledge on stellar CME parameters a logical approach is to deduce them from observations. Up to now only a handful of stellar CMEs has been detected. This poses the question if CMEs are a rare phenomenon on stars? The majority of the stellar CME detections from literature have used the signature of stellar material being ejected into the stellar astrosphere, which is Doppler-shifted flux seen either in emission or absorption in the blue wings of Balmer lines, as in many cases the CME core is a filament with a strong signal in the Balmer lines. Moreover, nearly all of the so far known stellar CMEs were detected by chance, except for the study by Guenther \& Emerson (1997). Therefore our team initiated search programs for stellar CMEs (Leitzinger et al. (2014), Korhonen et al. (2017)). The data we analyzed to date did not show signatures of CMEs, but we are continuing with those programs and developed in addition the idea of a flare alert system, which is based on long-term photometric monitoring at Observatory Lustbühel Graz (OLG) of a high number of late-type main-sequence stars. If a flare is detected, an alert is sent to involved observatories doing then the spectroscopic follow up. In this way up to several thousands of stars can be investigated simultaneously.

To estimate the expected occurrence rates of CMEs on other stars, one may extrapolate relations between flares and CMEs from the Sun and combine them with stellar flare rates. This has been done recently by Aarnio et al. (2012), Drake et al. (2013), and Odert et al. (2017). Thereby it is possible to compare the existing stellar CME detections 


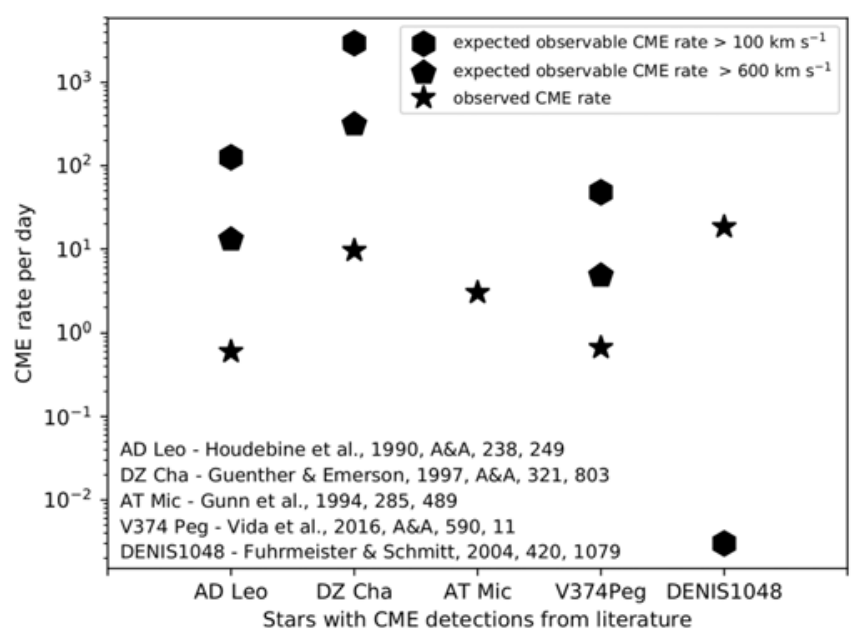

Figure 1. Expected observable (pentagon and hexagon symbols) versus observed CME rates from literature (black star symbols). The pentagon and hexagon symbols correspond to expected observable CME rates for CMEs with $>100 \mathrm{~km} \mathrm{~s}^{-1}$ and $>600 \mathrm{~km} \mathrm{~s}^{-1}$ ( $\mathrm{v}_{\text {los }}>\mathrm{v}_{\text {esc }}$ ), respectively. The expected observable CME rates are calculated with the CME model by Odert et al. (2017) and its updated version (Odert et al., in preparation). All expected observable CME rates exceed the observed ones except for the DENIS1048 event. This is mainly caused by the fact that this probable slow stellar CME event was detected in only $1.3 \mathrm{~h}$ of total observing time and extrapolation of the model to an M9 star is in this case not representative.

with the expected CME rates. As for now, we know three events from literature which have a high probability to represent indeed CMEs, because the projected velocities of the events were larger than the escape velocity of the corresponding stars. Two further events showed lower velocities, but have the typical signature of stellar CMEs. In Fig. 1 we show the results of this comparison. As one can clearly see the observed CME rates are far below the predicted rates. This might be related to the following reasons: (a) Model ingredients: Extrapolating the solar flare-CME relations (association rate, CME massflare energy relation, CME velocity-flare energy relation) to stars with higher activity levels and different spectral types may lead to overestimations. Observations are needed to confirm if these relations hold or break down for other parameter ranges. (b) Limitations of the observational methodology: Only the most luminous (i.e. massive) CMEs may be observable in optical spectra as distinct extra-emissions/absorptions. Solar erupting filaments can be seen up to several solar radii - those would be detectable with the Doppler method if the filament is not dissolved too quickly into the wind. The duration of CMEs may thus be too short because of signal dilution during propagation and expansion.(c) Physics: Not all CMEs may escape on other stars because of a strong overlaying magnetic field (Alvarado-Gomez et al. (2018)).

We want to conclude here that besides the reasons given above probably alternative methods need to be developed to study CMEs on stars.

\section{References}

Aarnio, A. N., Matt, S. P., \& Stassun, K. G 2012, ApJ, 760, 9

Alvarado-Gomez, J. D., Drake, J. J., Cohen, O. et al. 2018, ApJ, 862, 93

Drake, J. J., Cohen, O., Yashiro, S., \& Gopalswamy, N. 2013, ApJ, 764, 170

Guenther, E. W. \& Emerson, J. P. 1997, A\& A, 321, 803

Korhonen, H., Vida, K., Leitzinger, M., Odert, P., \& Kovacs 2017, IAUS, 328, 198

Leitzinger, M., Odert, P., Greimel, R., et al. 2014, MNRAS, 443, 898

Odert, P., Leitzinger, M., Hanslmeier, A., \& Lammer, H. 2017, MNRAS,472, 876 